\title{
Molecular Dynamics Simulations of the Thermal Conductivity of Silicon-Germanium and Silicon-Germanium-Tin Alloys
}

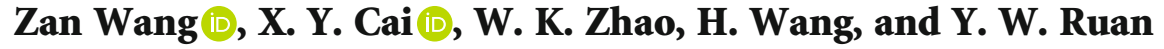 \\ School of Mechanical and Electrical Engineering, Henan University of Technology, Zhengzhou 450007, China \\ Correspondence should be addressed to Zan Wang; truewz@163.com
}

Received 16 December 2020; Revised 5 April 2021; Accepted 25 April 2021; Published 6 May 2021

Academic Editor: Hassan Karimi-Maleh

Copyright (c) 2021 Zan Wang et al. This is an open access article distributed under the Creative Commons Attribution License, which permits unrestricted use, distribution, and reproduction in any medium, provided the original work is properly cited.

\begin{abstract}
In this work, we investigate the thermal conductivity properties of $\mathrm{Si}_{1-x} \mathrm{Ge}_{x}$ and $\mathrm{Si}_{0.8} \mathrm{Ge}_{0} \mathrm{Sn}_{2 y}$ alloys. The equilibrium molecular dynamics (EMD) is employed to calculate the thermal conductivities of $\mathrm{Si}_{1-x} \mathrm{Ge}_{x}$ alloys when $x$ is different at temperatures ranging from $100 \mathrm{~K}$ to $1100 \mathrm{~K}$. Then nonequilibrium molecular dynamics (NEMD) is used to study the relationships between $y$ and the thermal conductivities of $\mathrm{Si}_{0.8} \mathrm{Ge}_{0.2} \mathrm{Sn}_{2 y}$ alloys. In this paper, $\mathrm{Ge}$ atoms are randomly doped, and tin atoms are doped in three distributing ways: random doping, complete doping, and bridge doping. The results show that the thermal conductivities of $\mathrm{Si}_{1-x} \mathrm{Ge}_{x}$ alloys decrease first, then increase with the rise of $x$, and reach the lowest value when $x$ changes from 0.4 to 0.5 . No matter what the value of $x$ is, the thermal conductivities of $\mathrm{Si}_{1-x} \mathrm{Ge}_{x}$ alloys decrease with the increase of temperature. Thermal conductivities of $\mathrm{Si}_{0.8} \mathrm{Ge}_{0.2}$ alloys can be significantly inhibited by doping an appropriate number of $\mathrm{Sn}$ atoms. For the random doping model, thermal conductivities of $\mathrm{Si}_{0.8} \mathrm{Ge}_{0.2} \mathrm{Sn}_{y}$ alloys approach the lowest level when $y$ is 0.10 . Whether it is complete doping or bridge doping, thermal conductivities decrease with the increase of the number of doped layers. In addition, in the bridge doping model, both the number of Sn atoms in the [001] direction and the penetration distance of Sn atoms strongly influence thermal conductivities. The thermal conductivities of $\mathrm{Si}_{0.8} \mathrm{Ge}_{0.2} \mathrm{Sn}_{y}$ alloys are positively associated with the number of $\mathrm{Sn}$ atoms in the [001] direction and the penetration distance of $\mathrm{Sn}$ atoms.
\end{abstract}

\section{Introduction}

Attention to metal nanomaterials and their applications can be creating a new approach in science [1-3]. Besides thermoelectric materials, nanomaterials are widely used to modify properties of composite materials, such as pharmaceutical material [4] and electrolyte material [4-12]. Due to the high thermal conductivity of $\mathrm{Si}$ and Ge, they are not suitable to be used as thermoelectric materials alone. However, when the two materials are completely alloyed, the thermal conductivities of the alloys are significantly lower than that of the two materials themselves. Researchers usually use $\mathrm{Si}$ as matrix materials to prepare $\mathrm{SiGe}$ alloys by doping Ge into $\mathrm{Si}$. In SiGe alloys, by controlling the ratio of $\mathrm{Ge}$ to $\mathrm{Si}$, the thermal conductivities can be modulated artificially. Since SiGe alloys have the advantages of good stability, high melting point, and strong oxidation resistance, they are widely used in spacecraft radioisotope batteries, power generators for micro devices, etc. To improve their thermoelectric performance, it is necessary to reduce thermal conductivities of SiGe alloys. Doping other elements into SiGe alloys is an effective way to reduce thermal conductivities. Because of the limitations of preparation methods and experimental measurements, several numerical tools including lattice dynamics, molecular dynamics, the Boltzmann transport equation, and the Monte Carlo method are often employed to investigate the behaviors of heat transfer in nanoscale materials.

Molecular dynamics (MD) is a feasible micro-nanoscale research method based on classical Newtonian mechanics $[13,14]$ and is suitable for the study of heat conduction of SiGe alloys with dopants at the micro-nanoscale [15-23]. $\mathrm{MD}$ can be divided into equilibrium molecular dynamics (EMD) and nonequilibrium molecular dynamics (NEMD). Basing on the linear response theory, EMD is good at the 
system in equilibrium state. The latter is fit for the nonequilibrium state and can be further categorized into homogeneous and inhomogeneous modes in terms of the application conditions of periodic boundary.

In recent years, some researches attempted to dope Sn into $\mathrm{SiGe}$ alloys to reduce the thermal conductivities of the alloys [24]. In order to verify the feasibility of this method and clarify the suppression mechanism of thermal conductivities in SiGe alloys, we applied LAMMPS (large-scale atomic/molecular massively parallel simulator) to calculate the thermal conductivities of $\mathrm{Si}_{1-x} \mathrm{Ge}_{x}$ and $\mathrm{Si}_{0.8} \mathrm{Ge}_{0.2} \mathrm{Sn}_{y}$ alloys ( $x$ is the ratio of the number of Ge atoms to the total number of atoms in the alloys, and $y$ is the ratio of the number of Sn atoms to the sum of the number of Si and Ge atoms) by adopting the Stillinger-Weber (SW) multibody potential as follows [25]:

$$
\begin{aligned}
U= & U_{2}\left(r_{i j}\right)+U_{3}\left(r_{i}, r_{j}, r_{k}\right) \\
= & \varepsilon f_{2}\left(r_{i j} / \sigma\right)+\varepsilon f_{3}\left(r_{i} / \sigma, r_{j} / \sigma, r_{k} / \sigma\right), \\
f_{2}\left(r_{i j}\right)= & \frac{A}{r_{i j}}\left[\frac{4 B}{r_{i j}^{5}}+\left(\frac{B}{r_{i j}^{4}}-1\right) /\left(r_{i j}-r_{c}\right)^{2}\right] \exp \\
& \cdot\left(\frac{1}{r_{i j}-r_{c}}\right) r_{i j}, \\
f_{3}\left(r_{i}, r_{j}, r_{k}\right)= & h\left(r_{i j}, r_{i k}\right)+h\left(r_{j i}, r_{j k}\right)+h\left(r_{k i}, r_{k j}\right), \\
h\left(r_{i j}, r_{i k}\right)= & \lambda \exp \left(\frac{\gamma}{r_{i j}-r_{c}}+\frac{\gamma}{r_{i k}-r_{c}}\right) \\
& \cdot\left(\cos \theta_{j i k}+\frac{1}{3}\right)^{2},
\end{aligned}
$$

where $U_{2}$ and $U_{3}$ are the two-body interaction potential and three-body interaction potential included in the SW potential, $f_{2}$ is the dimensionless two-body interaction potential and $f_{3}$ is the dimensionless three-body interaction potential, $r_{i j}$ is the distance between atoms $i$ and $j, r_{c}$ is the truncation radius, and $\theta_{j i k}$ is the angle between the vectors $r_{i j}$ and $r_{i k}$. Besides, $A, B, \sigma, \varepsilon, \lambda$, and $\gamma$ are the potential parameters.

\section{Molecular Dynamics Simulations of the Thermal Conductivity of SiGe Alloys and SiGeSn Alloys}

For SiGe alloys, the parameters required to simulate the thermal conductivities of SiGe alloys are given in Table 1 [26].

The lattice constants of $\mathrm{Si}_{1-x} \mathrm{Ge}_{x}$ alloys can be calculated by the Vegard law, and the expression is as follows:

$$
a_{\mathrm{SiGe}}=(1-x) a_{\mathrm{Si}}+x a_{\mathrm{Ge}} .
$$

In simulations, $\mathrm{Ge}$ atoms were mixed into $\mathrm{Si}$ atoms in a random doping distribution. Periodic boundary conditions were set in the $x, y$, and $z$ directions. The simulation scale was $8 L \times 4 L \times 4 L, L$ equals to the thickness of 1 unit cell (UC). There were 1024 atoms in the microcanonical (NVE) ensemble system. The integral algorithm selected was the Velocity-Verlet algorithm [27], and the integration step length was $0.76 \mathrm{fs}$. The total number of steps was $2.5 \times 10^{6}$, and the total simulation time was $1.90 \mathrm{~ns}$. The system first ran $5 \times 10^{5}$ steps to realize thermal balance and then ran $2.0 \times 10^{6}$ steps to count the instantaneous heat flow. The EMD method was applied to calculate thermal conductivities in [100] direction by

$$
\kappa=\frac{1}{3 V k_{B} T^{2}} \int_{0}^{\infty}\langle J(t) \cdot J(0)\rangle d t,
$$

where $k_{B}$ is the Boltzmann constant and $J$ is the effective heat flow.

As to SiGeSn alloys, the values of the SW potential parameters for $\mathrm{Sn}-\mathrm{Sn}, \mathrm{Sn}-\mathrm{Ge}$, and $\mathrm{Sn}-\mathrm{Si}$ interactions in SiGeSn were determined by using a force-matching method based on the density functional theory (DFT) calculations [28], as shown in Table 2.

For $\mathrm{Si}_{x} \mathrm{Ge}_{1-x-y} \mathrm{Sn}_{y}$, the lattice constant $a_{\mathrm{Six} \mathrm{Ge} 1-x-y \mathrm{Sn} y}$ can be calculated by the following formula [29]:

$$
\begin{aligned}
a_{\mathrm{SiGeSn}}= & a_{\mathrm{Ge}}+\Delta_{\mathrm{SiGe}} \times x+\theta_{\mathrm{GeSi}} x(1-x) \\
& +\Delta_{\mathrm{SnGe}} \times y+\theta_{\mathrm{GeSn}} y(1-y),
\end{aligned}
$$

where $\Delta_{\mathrm{SiGe}}\left(\Delta_{\mathrm{SnGe}}\right)=a_{\mathrm{Si}}-a_{\mathrm{Ge}}\left(a_{\mathrm{Sn}}-a_{\mathrm{Ge}}\right), \quad \theta_{\mathrm{SiGe}}=-0.026$, and $\theta_{\mathrm{GeSn}}=0.166$. After transformation, the expression of $a_{\mathrm{Si} 0.8 \mathrm{Ge} 0.2 \mathrm{Sn} y}$ is obtained as follows:

$$
\begin{aligned}
a_{\mathrm{Si} 0.8 \mathrm{Ge} 0.2 \mathrm{Sn} y}= & a_{\mathrm{Ge}}+0.8 \times \Delta_{\mathrm{SiGe}}+0.8 \times(0.2+y) \\
& \times \theta_{\mathrm{GeSi}}+\Delta_{\mathrm{SnGe}} \times y+\theta_{\mathrm{GeSn}} \times y .
\end{aligned}
$$

In this work, the NEMD method was used to simulate the thermal conductivities of $\mathrm{Si}_{0.8} \mathrm{Ge}_{0.2} \mathrm{Sn}_{y}$ in [100] direction. Periodic boundary conditions were set in $y$ and $z$ directions. Heat flow was applied in the $\mathrm{x}$ direction. Thermal insulation wall, heat source, intermediate layer, and cold source were installed. The length of the wall was $2 \times$ UC. The length of the heat source and the cold source was both $3 \times \mathrm{UC}$. The length of the intermediate layer was set as $20 \times \mathrm{UC}$. We used the Velocity-Verlet algorithm as the integration algorithm and set the time step as $0.5 \mathrm{fs}$, which is less than $1 / 10$ of the shortest vibration period in this work. In addition, Sn atoms were doped by random distribution, complete doping, and bridge doping, respectively, and the classical Boltzmann statistics was used to calculate the temperature of the $i$ th layer in this work by

$$
\frac{1}{2}\left\langle\sum_{i=1}^{N_{i}} m_{i} v_{i}^{2}\right\rangle=\frac{3}{2} N_{i} k_{B} T_{i, \mathrm{MD}}
$$

where $N_{i}$ is the number of particles in the $i$ th layer and $T_{i, \mathrm{MD}}$ is the local temperature of the $i$ th layer. After the value of heat 
TABLe 1: Interatomic simulation parameters of Si-Si, Ge-Ge, and Si-Ge.

\begin{tabular}{lccccccrrr}
\hline- & $m$ & $\varepsilon(e V)$ & $\begin{array}{c}\sigma \\
\left(10^{-10} \mathrm{~m}\right)\end{array}$ & $\begin{array}{c}a \\
\left(10^{-10} \mathrm{~m}\right)\end{array}$ & $A$ & $B$ & $\lambda$ & $\gamma$ \\
\hline $\mathrm{Si}-\mathrm{Si}$ & 4.66 & 1.41992 & 2.105 & 5.431 & 7.05 & 0.6022 & 29.53 & 1.20 \\
$\mathrm{Ge}-\mathrm{Ge}$ & 12.05 & 1.30665 & 2.221 & 5.658 & 7.05 & 0.6022 & 24.63 & 1.20 \\
$\mathrm{Si}-\mathrm{Ge}$ & - & 1.45930 & 2.141 & - & 7.05 & 0.6022 & 31.08 & 1.34 & 1.84 \\
\hline
\end{tabular}

TABLE 2: Interatomic simulation parameters of Si-Sn, Ge-Sn, and Sn-Sn.

\begin{tabular}{lccccccc}
\hline- & $\sigma(10-10 m)$ & $\varepsilon(e V)$ & $\lambda$ & $r_{c}$ & $\gamma$ & $A$ & $B$ \\
\hline Sn-Sn & 2.5681 & 1.2351 & 19.0031 & 1.8 & 1.2 & 7.05 & 0.6022 \\
Sn-Si & 2.2617 & 1.6435 & 8.7433 & 1.9172 & 1.1 & 7.05 & 0.6022 \\
Ge-Sn & 2.3313 & 1.5592 & 11.9601 & 1.8894 & 1.1 & 7.05 & 0.6022 \\
\hline
\end{tabular}

flow and temperature gradient are obtained, the Fourier law can be used to solve the thermal conductivity as follows:

$$
\lambda=-\frac{\Phi}{A \times \nabla T}
$$

where $\lambda$ is the thermal conductivity, $\Phi$ is the value of heat flow, $A$ is the cross-sectional area perpendicular to the direction of heat flow, and $\nabla T$ is the temperature gradient.

\section{Results and Discussion}

So, to verify the correctness of our model, we studied the thermal conductivities of SiGe alloys by changing the proportion of doped $\mathrm{Ge}$ atoms at $300 \mathrm{~K}$ in Figure 1. It can be seen that the thermal conductivities of SiGe show parabolic trends with the increasing proportion of Ge atoms. When $x$ locates at $0.1 \sim 0.4$, the thermal conductivity decreases with the increase of $x$. This is because the atomic mass and lattice constant of $\mathrm{Si}$ and $\mathrm{Ge}$ atoms are quite different, which exacerbates the defect scattering of short-wave phonons by lattice point defects. However, when $x$ increases from 0.5 to 1.0, the thermal conductivities increase too, which suggests that the enhancement of thermal conduction of Ge atoms surpasses the hindrance of lattice point defect. In Figure 1, the solid squares are the results of the EMD simulation similar to the experimental results [30] marked as solid circles.

The thermal conductivities of SiGe with different temperatures were calculated in Figure 2. The results show the thermal conductivities of $\mathrm{Si}_{0.8} \mathrm{Ge}_{0.2}$ decrease significantly with the increase of temperatures and reach a flat level at about $1000 \mathrm{~K}$. The reason for the decrease of thermal conductivities is that the number of phonons with high frequency increases at high temperatures giving rise to the increase of phononphonon inelastic scattering processes.

Subsequently, we evaluated the thermal conductivities of $\mathrm{Si}_{0.8} \mathrm{Ge}_{0.2} \mathrm{Sn}_{y} \mathrm{Si}_{0.8} \mathrm{Ge}_{0.2} \mathrm{Sn}_{y}$ by varying the proportion of $\mathrm{Sn}$ atoms at $300 \mathrm{~K}$, as illustrated in Figure 3. The thermal conductivities of the random doping model decrease first, then increase with the increase of the proportion of $\mathrm{Sn}$ atoms, and reach the lowest value when $y$ equals 0.10 . This principle

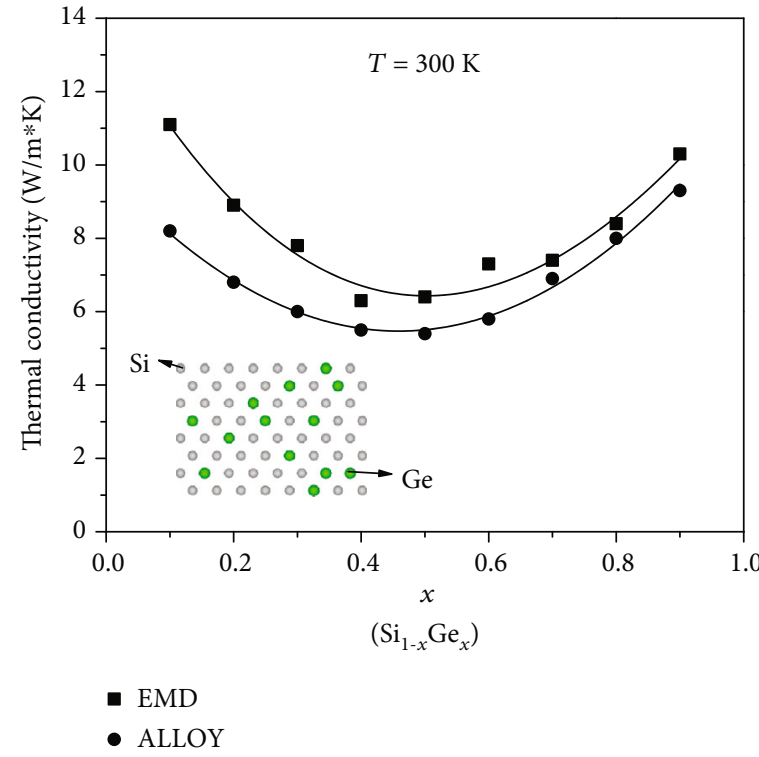

FIgURE 1: Variation of the thermal conductivities of $\mathrm{Si}_{1-x} \mathrm{Ge}_{x}$ at $300 \mathrm{~K}$. The inset is the doping schematic diagram of Ge atoms.

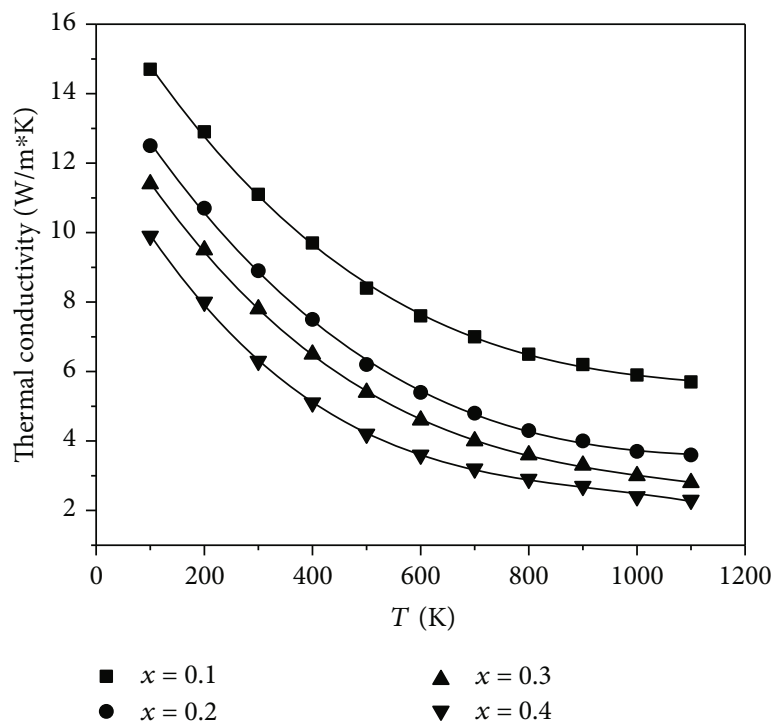

FIgURE 2: Thermal conductivities of $\mathrm{Si}_{1-x} \mathrm{Ge}_{x}$ at different temperatures.

is the same as that shown in Figure 1. Because of the doping of Sn atoms, the lattice is distorted by a large number of point defects, which intensifies the scattering rate of short-wave phonons and lowers the thermal conductivity. But when the proportion of $\mathrm{Sn}$ atoms reaches a certain degree, its 


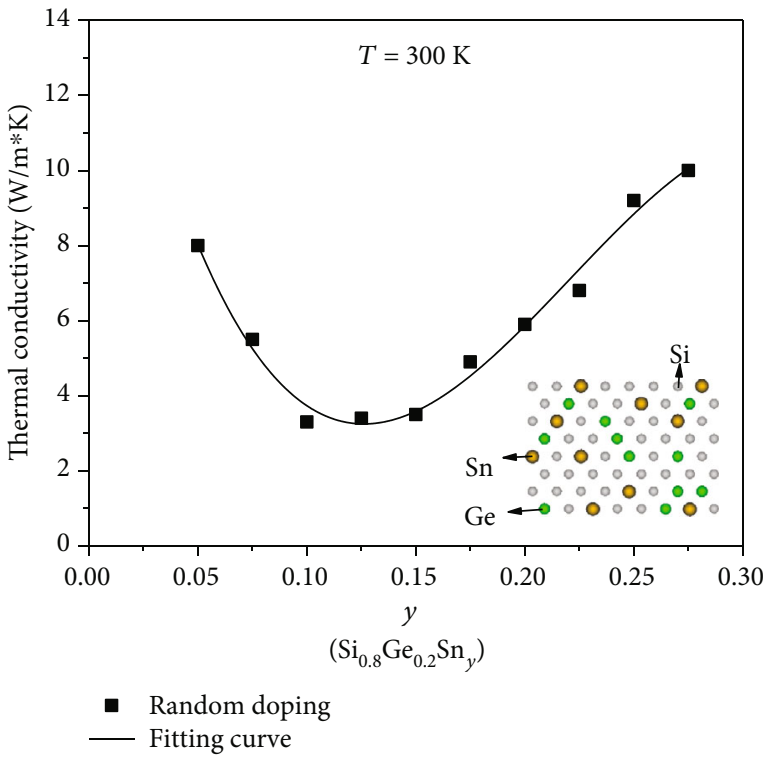

FIGURE 3: Variation of the thermal conductivities of $\mathrm{Si}_{0.8} \mathrm{Ge}_{0.2} \mathrm{Sn}_{y}$ at $300 \mathrm{~K}$. The inset is the schematic diagram of random doping model.

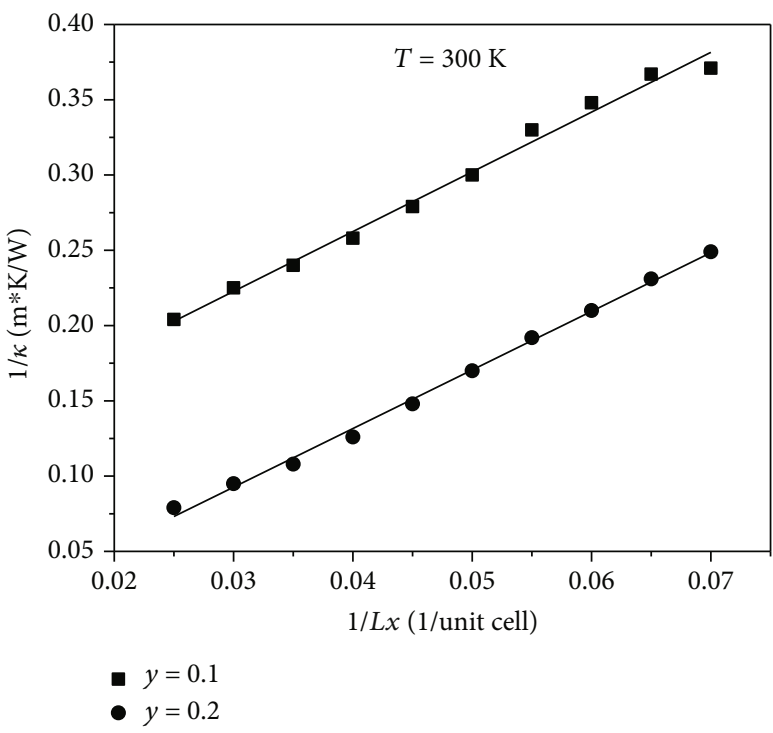

Figure 4: Relationships between thermal conductivities and the length of the intermediate layer.

enhancement of heat transfer becomes predominantly, leading to a gradual increase of the thermal conductivities.

The relationships between the thermal conductivities of $\mathrm{Si}_{0.8} \mathrm{Ge}_{0.2} \mathrm{Sn}_{y}$ and the length of the intermediate layer at $300 \mathrm{~K}$ are shown in Figure 4 . The Sn atoms were doped by random doping. The reciprocal of the thermal conductivities is approximately positively correlated with that of the length of the intermediate layer, and the thermal conductivities increase with the increase of the length of the intermediate layer. The results show an obvious size effect as the reported results [31].

The simulations of the thermal conductivities changing with temperatures under different number of doping layers in complete doping model are shown in Figure 5. Although

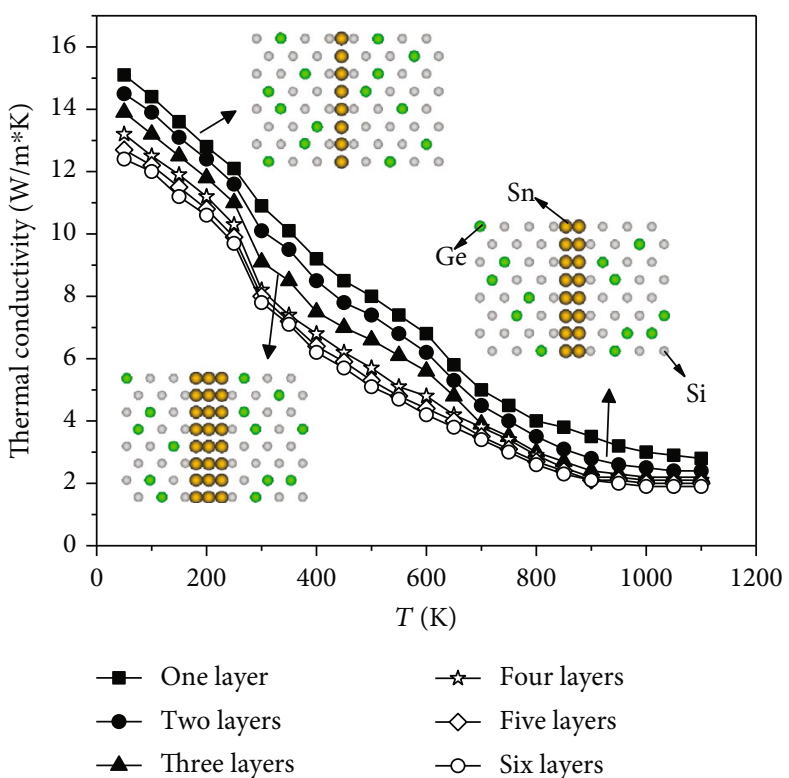

FIgURE 5: Thermal conductivities of $\mathrm{Si}_{0.8} \mathrm{Ge}_{0.2} \mathrm{Sn}_{y}$ with different doping layers in complete doping model. The insets are schematic diagrams of complete doping model.

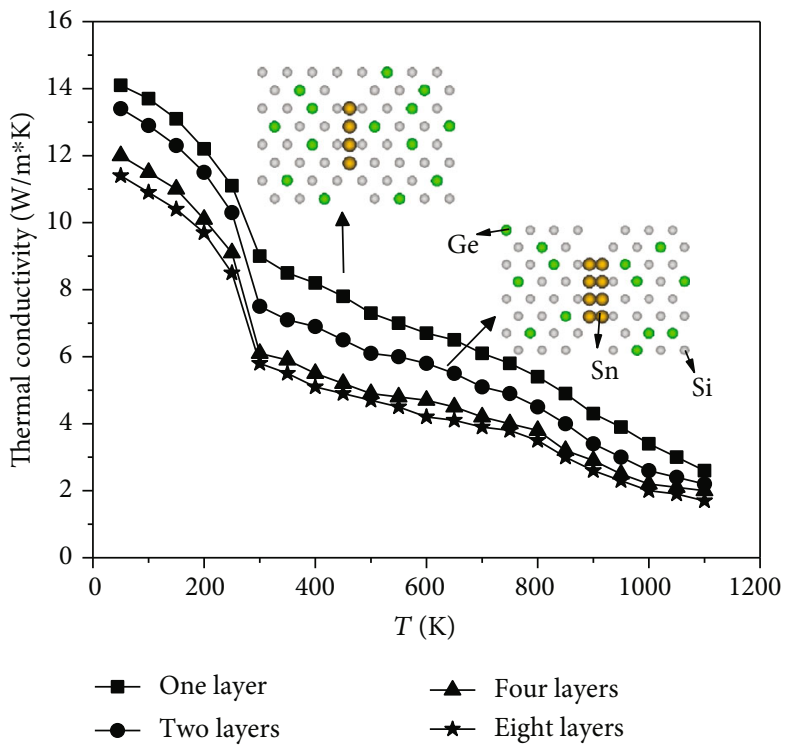

FIgure 6: Thermal conductivities of $\mathrm{Si}_{0.8} \mathrm{Ge}_{0.2} \mathrm{Sn}_{y}$ with different doping layers in bridge doping model. The insets are the schematic diagrams of the bridge doping model.

the number of layers of doped $\mathrm{Sn}$ atoms is different, the thermal conductivities decrease as the temperatures increase. This is still because the rate of the Umklapp (U) scattering process increases at high temperatures, and so, the phonon mean free path (MFP) decreases. When the number of doped layers $n \leq 4$, the thermal conductivities decrease with the increase of the number of doped layers. As $n>4$, the decreasing range becomes smaller, and the simulation results tend to be stable. In Figure 6, the results show that when the bridge doping model is used, the $U$ process still reduces the thermal conductivities, and finally, the thermal conductivities become 


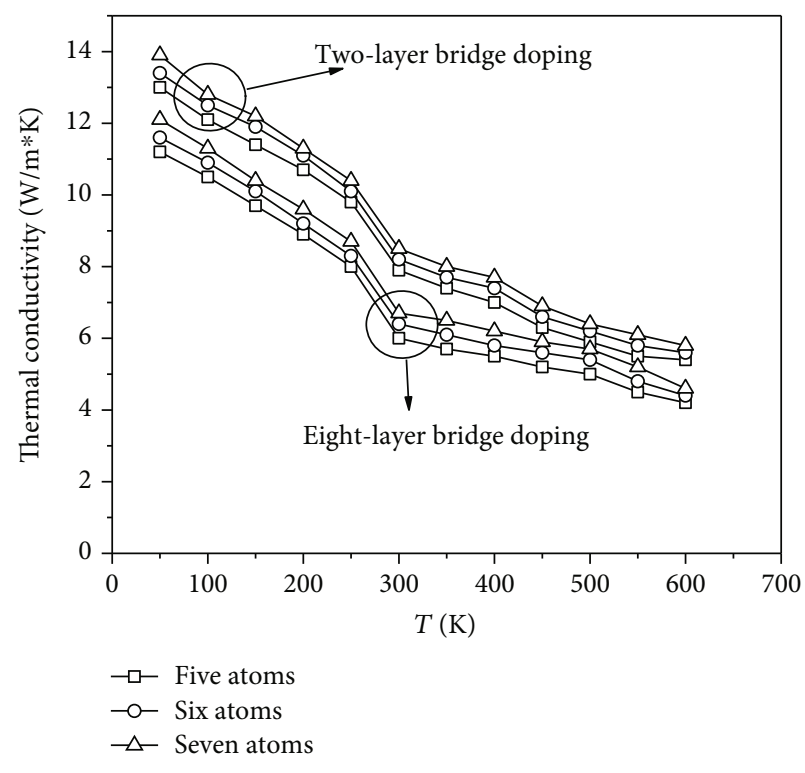

Figure 7: Thermal conductivities with different atomic numbers in the [001] direction.

a constant value at a certain temperature. When the number of doped layers $n \leq 4$, the increase of the number of doped layers will lead to an effective decrease of thermal conductivities, but when $n>4$, the increase of the number of doped layers will not cause an obvious change of thermal conductivities. In Figures 5 and 6, the thermal conductivities will have a sharp decreasing trend between $250 \mathrm{~K}$ and $300 \mathrm{~K}$. Moreover, when the temperature is higher than $700 \mathrm{~K}$, the curves of thermal conductivities begin to converge. The reason may be explained by the fact that the simulated temperature is higher than the melting point of Sn, and the influence of the number of doped layers of Sn atoms on the thermal conductivities becomes weaker than ever.

The decrease of thermal conductivities of the two doping modes with the increase of the number of doping layers is presumably due to the doped $\mathrm{Sn}$ atoms regularly arranged, and the surrounding lattice structure is torn to form grain boundaries, which intensifies the boundary scattering of phonons and reduces the phonon MFP, leading to the decrease of thermal conductivities. But while the number of layers increases to a special value, the effect of boundary scattering is saturated, and most of the phonons pass through the boundary and continue to transfer heat after overcoming the thermal resistance. Comparing the two doping methods, it is not difficult to find that the thermal conductivities of bridge doping are lower than that of complete doping with the same number of doped layers. The reason for this phenomenon may be attributed to that bridge doping narrows the propagation path of phonons and limits their MFP.

Figure 7 shows the results of thermal conductivities with different atomic numbers in the [001] direction when the number of layers of bridge doping is two and eight. It can be seen that no matter how many layers of Sn atoms are doped, the thermal conductivities increase as the number of atoms increases, which further confirms the above conclu-

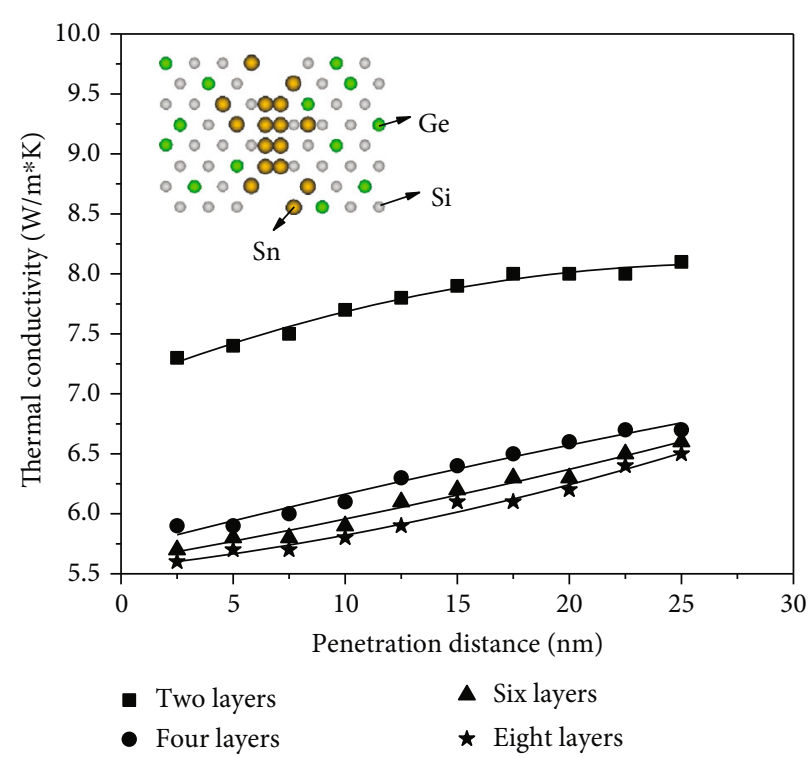

FIgURE 8: The influence of penetration distance on thermal conductivities.

sion that the thermal conductivities of bridge doping are lower than that of complete doping.

However, when bridge doping model is applied in this simulation, it was found that $\mathrm{Sn}$ atoms will penetrate to both sides and cause a certain change in the values of thermal conductivities. Therefore, we have investigated the dependence of the penetration distance of Sn atoms on the thermal conductivities under different doping layers at $300 \mathrm{~K}$. The simulations are shown in Figure 8, which shows the results of twolayer, four-layer, and eight-layer bridge doping models separately, assuming that the penetrative Sn atoms are randomly distributed. It was found the thermal conductivities will increase slowly with the increase of the penetration distance. The reason may be that the scattering effect of point defects generated by random doping on phonons is weaker than that of boundary scattering on phonons.

\section{Conclusions}

In this work, the MD method is applied to investigate the thermal conduction properties of $\mathrm{Si}_{1-x} \mathrm{Ge}_{x}$ alloys and $\mathrm{Si}_{0.8}$ $\mathrm{Ge}_{0.2} \mathrm{Sn}_{y} \mathrm{Si}_{0.8} \mathrm{Ge}_{0.2} \mathrm{Sn}_{y}$ alloys. Some calculations in our work are performed below the Debye temperature of the material. In the simulations of $\mathrm{Si}_{1-x} \mathrm{Ge}_{x}$ alloys, Ge atoms are doped randomly. At $T=300 \mathrm{~K}$, the thermal conductivities of $\mathrm{Si}_{1-x}$ $\mathrm{Ge}_{x}$ reach the minimum value when $x$ locates at 0.4 to 0.5 . And the thermal conductivities decrease with the increase of temperature and tend to be stable at about $1100 \mathrm{~K}$. Further, we investigate the thermal features of alloys with three $\mathrm{Sn}$ doping modes as random doping, complete doping, and bridge doping. The results show that in random doping mode at $300 \mathrm{~K}$, the thermal conductivities of $\mathrm{Si}_{0.8} \mathrm{Ge}_{0.2} \mathrm{Sn}_{y}$ reach the minimum while $y$ is about 0.1 . Compared with the thermal conductivities of $\mathrm{Si}_{0.8} \mathrm{Ge}_{0.2}$ alloys, the thermal conductivities after doping with $\mathrm{Sn}$ atoms are significantly reduced, 
indicating that it is feasible to reduce the thermal conductivities by doping an appropriate amount of $\mathrm{Sn}$ atoms in actual experiments. In complete doping mode and bridge doping mode at $300 \mathrm{~K}$, when the number of doped layers $n \leq 4$, the thermal conductivities decrease significantly with the increase of $n$. When $n>4$, the thermal conductivities hardly change with the increase of $n$. Besides, when $\mathrm{Sn}$ atoms are doped by bridge doping, the more $\mathrm{Sn}$ atoms in the [001] direction and the farther Sn atoms penetrate, the higher the thermal conductivities will be.

\section{Data Availability}

The data used to support the findings of this study are available from the corresponding author upon request.

\section{Disclosure}

I confirm that this manuscript is the author's original work. The article has been written by the stated authors who are all aware of its content and approve its submission. The article has not been published previously. The article is not under consideration for publication elsewhere. If accepted, the article will not be published elsewhere in the same form, in any language, without the written consent of the publisher.

\section{Conflicts of Interest}

No conflict of interest exists.

\section{Acknowledgments}

This work was supported by the National Natural Science Foundation of China (51106043), the basic research project of Henan Provincial Department of Education Science and Technology (13A470176, 2014GGJS-059), and the special funding project of basic scientific research of Henan University of Technology (2017QNJH26), the opening project of National Facility for Translational Medicine (Shanghai).

\section{References}

[1] H. Karimi-Maleh, M. L. Yola, N. Atar et al., "A novel detection method for organophosphorus insecticide fenamiphos: molecularly imprinted electrochemical sensor based on core-shell $\mathrm{Co}_{3} \mathrm{O}_{4} @$ MOF-74 nanocomposite," Journal of Colloid and Interface Science, vol. 592, pp. 174-185, 2021.

[2] M. Morteza, A. Z. Hassan, and K. M. Hassan, "A sensitive electroanalytical sensor amplified with $\mathrm{Pd}-\mathrm{ZnO}$ nanoparticle for determination of Sunset Yellow in real samples," Eurasian Chemical Communications, vol. 2, no. 7, pp. 760-770, 2020.

[3] P. Naderi Asrami, P. Aberoomand Azar, M. Saber Tehrani, and S. A. Mozaffari, "Glucose oxidase/nano-ZnO/thin film deposit FTO as an innovative clinical transducer: a sensitive glucose biosensor," Frontiers in Chemistry, vol. 8, article 00503, 2020.

[4] M. Nodehi, M. Baghayeri, R. Behazin, and H. Veisi, "Electrochemical aptasensor of bisphenol A constructed based on 3D mesoporous structural SBA-15-Met with a thin layer of gold nanoparticles," Microchemical Journal, vol. 162, article 105825, 2021.
[5] M. Ghanei-Motlagh and M. Baghayeri, "Determination of trace $\mathrm{Tl}$ (I) by differential pulse anodic stripping voltammetry using a novel modified carbon paste electrode," Journal of The Electrochemical Society, vol. 167, no. 6, article 066508, 2020.

[6] M. Nodehi, M. Baghayeri, R. Ansari, and H. Veisi, "Electrochemical quantification of $17 \alpha$ - ethinylestradiol in biological samples using a $\mathrm{Au} / \mathrm{Fe}_{3} \mathrm{O}_{4} @ \mathrm{TA} / \mathrm{MWNT} / \mathrm{GCE}$ sensor," Materials Chemistry and Physics, vol. 244, article 122687, 2020.

[7] M. Baghayeri, M. Ghanei-Motlagh, R. Tayebee, M. Fayazi, and F. Narenji, "Application of graphene/zinc-based metal-organic framework nanocomposite for electrochemical sensing of As(III) in water resources," Analytica Chimica Acta, vol. 1099, pp. 60-67, 2020.

[8] M. Rouhi, M. M. Lakouraj, and M. Baghayeri, "Low band gap conductive copolymer of thiophene withp-phenylenediamine and its magnetic nanocomposite: synthesis, characterization and biosensing activity," Polymer Composites, vol. 40, no. 3, pp. 1034-1042, 2019.

[9] M. Ghanei-Motlagh, M. A. Taher, M. Fayazi, M. Baghayeri, and A. R. Hosseinifar, "Non-enzymatic amperometric sensing of hydrogen peroxide based on vanadium pentoxide nanostructures," Journal of The Electrochemical Society, vol. 166, no. 6, pp. B367-B372, 2019.

[10] M. Baghayeri, R. Ansari, M. Nodehi, I. Razavipanah, and H. Veisi, "Label-free electrochemical bisphenol A aptasensor based on designing and fabrication of a magnetic gold nanocomposite," Electroanalysis, vol. 30, no. 9, pp. 2160-2166, 2018.

[11] M. Baghayeri, R. Ansari, M. Nodehi, and H. Veisi, "Designing and fabrication of a novel gold nanocomposite structure: application in electrochemical sensing of bisphenol A," International Journal of Environmental Analytical Chemistry, vol. 98, no. 9, pp. 874-888, 2018.

[12] M. Baghayeri, R. Ansari, M. Nodehi, I. Razavipanah, and H. Veisi, "Voltammetric aptasensor for bisphenol A based on the use of a MWCNT/ $\mathrm{Fe}_{3} \mathrm{O}_{4} @$ gold nanocomposite," Microchimica Acta, vol. 185, no. 7, p. 320, 2018.

[13] B. J. Alder and T. E. Wainwright, "Phase transition for a hard sphere system," The Journal of Chemical Physics, vol. 27, no. 5, pp. 1208-1209, 1957.

[14] B. J. Alder and T. E. Wainwright, "Studies in molecular dynamics. I. General method," The Journal of Chemical Physics, vol. 31, no. 2, pp. 459-466, 1959.

[15] S. G. Volz and G. Chen, "Molecular dynamics simulation of thermal conductivity of silicon crystals," Physical Review B, vol. 61, no. 4, pp. 2651-2656, 2000.

[16] S. Huxtable and A. Abramson, "Thermal conductivity of Si/Ge and SiGe/SiGe superlattices," Applied Physics Letters, vol. 80, no. 10 , p. 1737, 2002.

[17] D. M. Rowe, V. S. Shukla, and N. Savvides, "Phonon scattering at grain boundaries in heavily doped fine-grained silicon- germanium alloys," Nature, vol. 290, no. 5809, pp. 765-766, 1981.

[18] X. B. Li and R. G. Yang, "Equilibrium molecular dynamics simulations for the thermal conductivity of $\mathrm{Si} / \mathrm{Ge}$ nanocomposites," Journal of Applied Physics, vol. 113, no. 10, article 104306, 2013.

[19] M. P. Alien, Computer Simulation of Liquids, Clarendon Press, Oxford, 1987.

[20] Y. W. Yang, X. J. Liu, and J. P. Yang, "Nonequilibrium molecular dynamics simulation for size effects on thermal conductivity of Si nanostructures," Molecular Simulation, vol. 34, no. 1, pp. 51-56, 2008. 
[21] F. Müller-Plathe, "A simple nonequilibrium molecular dynamics method for calculating the thermal conductivity," The Journal of Chemical Physics, vol. 106, no. 14, pp. 60826085, 1997.

[22] A. Maiti, G. D. Mahan, and S. T. Pantelides, "Dynamical simulations of nonequilibrium processes - heat flow and the Kapitza resistance across grain boundaries," Solid State Communications, vol. 102, no. 7, pp. 517-521, 1997.

[23] S. Kotake and S. Wakuri, "Molecular dynamics study of heat conduction in solid materials," JSME International Journal Series B Fluids and Thermal Engineering, vol. 37, no. 1, pp. 103-108, 1994.

[24] S. N. Khatami and Z. Aksamija, "Lattice thermal conductivity of the binary and ternary group-IV alloys $\mathrm{Si}-\mathrm{Sn}, \mathrm{Ge}-\mathrm{Sn}$, and $\mathrm{Si}-$ Ge-Sn," Physical Review Applied, vol. 6, no. 1, article 014015, 2016.

[25] B. L. Holian and P. S. Lomdahl, "Plasticity induced by shock waves in nonequilibrium molecular-dynamics simulations," Science, vol. 280, no. 5372, pp. 2085-2088, 1998.

[26] Y. J. Lee and G. S. Hwang, "Microsegregation effects on the thermal conductivity of silicon-germanium alloys," Journal of Applied Physics, vol. 114, no. 17, article 174910, 2013.

[27] K. Tsuruta, A. Nakano, and R. K. Kalia, "Dynamics of consolidation and crack growth in nanocluster-assembled amorphous silicon nitride," Journal of the American Ceramic Society, vol. 81, p. 433, 1998.

[28] Y. J. Lee and G. S. Hwang, "Molecular dynamics investigation of the thermal conductivity of ternary silicon-germanium-tin alloys," Journal of Physics D: Applied Physics, vol. 50, no. 49, article 494001, 2017.

[29] P. Moontragoon, P. Pengpit, T. Burinprakhon et al., "Electronic properties calculation of $\mathrm{Ge}_{1-\mathrm{x}-\mathrm{y}} \mathrm{Si}_{\mathrm{x}} \mathrm{Sn}_{\mathrm{y}}$ ternary alloy and nanostructure," Journal of Non-Crystalline Solids, vol. 358, no. 17, pp. 2096-2098, 2012.

[30] M. S. Dresselhaus, G. Chen, M. Y. Tang et al., "New directions for low-dimensional thermoelectric materials," Advanced Materials, vol. 19, no. 8, pp. 1043-1053, 2007.

[31] J. W. Che, T. Çağın, W. Q. Deng, and W. A. Goddard III, "Thermal conductivity of diamond and related materials from molecular dynamics simulations," The Journal of Chemical Physics, vol. 113, no. 16, pp. 6888-6900, 2000. 